\title{
TURISMO AGROALIMENTARIO EN ÁREAS DE CULTIVO DE MANZANA EN LA REGIÓN SUR DE BRASIL
}

\author{
Fernando Goulart Rocha* \\ Instituto Federal de Santa Catarina
}

Antoni F. Tulla**

Universitat Autònoma de Barcelona

\section{RESUMEN}

En la Región Sur del Brasil, las experiencias de turismo agroalimentario en los municipios productores de manzana se caracterizan por el modesto aprovechamiento de la fruta como atractivo turístico. En esta sentido, el objetivo del artículo es presentar y discutir el marco actual del turismo agroalimentario de la manzana en la Región Sur de Brasil. La investigación contó con trabajo de campo y el estudio de la bibliografía pertinente en el tema. Para el análisis se recuperan los principios y contenido de la organización de los Sistemas Agroalimentarios Localizados y la consideración de las actividades accesorias como una estrategia de diversificación de la renta en la pequeña propiedad familiar. Las conclusiones del estudio son de que con el propósito de mejorar el turismo regional, es importante la concepción de un proyecto de desarrollo que contribuya positivamente hacia los sectores interesados en invertir en esta actividad. Por estos motivos, la cualificación profesional, la organización colectiva de los productores y procesadores, así como la participación del poder público, son imprescindibles para el éxito del turismo agroalimentario de la manzana en la Región Sur de Brasil.

Palabras clave: Turismo, planeación, manzana, agroalimentario, Brasil.

Recibido: 1 de septiembre de 2013

Devuelto para su revisión: 19 de mayo de 2014

Aceptado: 9 de julio de 2014

* Instituto Federal de Santa Catarina, Campus Florianópolis-Continente. Rua Quatorze de Julho, 150, Estreito, Florianópolis, SC, Brasil. CEP: 88075-010. E-mail: fernandogr@ifsc.edu.br

** Departamento de Geografía. Universidad Autónoma de Barcelona. Edificio B. 08193 Bellaterra. BARCELONA (España).E-mail: antoni.tulla@uab.cat 


\title{
Agro-food tourism in apple-growing areas in South Brazil
}

\begin{abstract}
In Brazil's South Region, experience of agro-food tourism in the apple-growing counties is characterized by the modest exploitation of the fruit as a tourist attraction. Objective: To present and discuss the current framework of agro-food tourism in the apple-growing areas of Brazil's South Region. Methodology: Our research included field studies and a review of the relevant literature. In our analysis we consulted the budgets of the Localized Agro-food Systems Organization, and we considered collateral activities as a strategy for diversifying the earnings of family smallholdings. Results: in order to boost local tourism, a development project making positive contributions to potential investors in the industry should be designed. Conclusion: Professional qualifications, the collective organization of the producers and processors, and state participation are all indispensable for the success of agro-food tourism in the apple-growing areas of Brazil's South Region.
\end{abstract}

Key words: Tourism, planning, apple, agro-food, Brazil.

\section{INTRODUCCIÓN}

En el 2011, Brasil contaba con la segunda área más grande de cultivo de manzanas de las Américas y ocupaba el noveno puesto a nivel mundial, en volumen de producción. (FAO, 2011). La fruticultura de clima templado está distribuida por 11 estados brasileños, siendo los tres estados de la Región Sur -Paraná, Santa Catarina y Rio Grande do Sul- que comprenden más del 70\% de la producción nacional. (Fachinello et al., 2011). Entre las frutas de clima templado están la uva, la manzana, el durazno, el caqui, la mora, el higo, la pera y el membrillo.

En relación al turismo en las áreas de fruticultura de clima templado en Brasil, se pueden destacar las actividades desarrolladas en las propiedades viticultoras. En dichas propiedades, en función de la inversión pública y privada que se ha aplicado en los últimos años, la actividad turística ganó impulso no solamente en relación a la calidad de la vid, sino, sobre todo, al mejor aprovechamiento de la fruta por medio de la producción de jugos y vinos finos de altitud. Asociados al cultivo de la uva y a la producción de vinos ha surgido la promoción de rutas turísticas vitivinícolas, como las del Vale dos Vinhedos, en el Estado de Rio Grande do Sul, y de la Serra Catarinense, en el Estado de Santa Catarina. (Lavandoski y Lanzer, 2008; Lossoy Pereira, 2012).

Sin embargo, el éxito del turismo vitivinícola es una excepción entre las variedades de frutas disponibles en esa región del país. En las propiedades productoras de manzanas no se ha dado una organización similar de productos y servicios turísticos, salvo experiencias recientes y de cobertura geográfica limitada. Por consiguiente, tales propiedades no han vislumbrado dicho aprovechamiento de la fruta para actividades que puedan diversificar la renta en la pequeña unidad familiar y agregar valor a la producción.

Frente a este escenario, el objetivo de este estudio es el de presentar y discutir el marco actual del turismo en áreas de cultivo de manzana en la Región Sur de Brasil, principal pro- 
ductora nacional de la fruta. En ese sentido, la investigación procura evaluar las experiencias actuales del turismo regional y señalar las posibilidades para la concepción de una propuesta de turismo agroalimentario centrado en presentar los productos y servicios turísticos de las propiedades rurales fruticultoras. En esta investigación se entiende por turismo agroalimentario, el turismo basado en realzar los Sistemas Agroalimentarios Localizados (SAL), es decir, las redes de «organización de producción y servicio asociadas a un territorio específico en virtud de sus características y funcionamiento.» (Díaz-Bautista, 2001: 15-16) ${ }^{1}$.

Bajo esos términos, para el análisis del contexto actual y perspectivas futuras del turismo agroalimentario regional, se recuperan los principios y contenido de la organización de los Sistemas Agroalimentarios Localizados (SAL) y la concepción de pluriactividad como estrategia de diversificación de la renta en la pequeña propiedad familiar. En la discusión de los resultados, a partir de los objetivos propuestos, se presentan las experiencias en vigor, las potencialidades y los elementos de planeación que deben ser incorporados a la actividad turística para que se pueda consolidar efectivamente en favor de los productores de manzana. Las conclusiones abordan los obstáculos actuales y las perspectivas del turismo local y regional.

\section{METODOLOGÍA}

El artículo es una reflexión personal de los autores acerca de la cuestión del turismo agroalimentario en las áreas de producción de manzana en Santa Catarina, fundamentada en un trabajo científico. Por lo tanto, la investigación contó con visitas tecnicas en el primero semestre de 2013 en las áreas destacadas y también lecturas de una bibliografía pertinente con el tema. Para los análisis fueran recuperados los principios y conceptos de la organización de los Sistemas Agroalimentarios Localizados (SAL) así como la concepción de las actividades accesorias entendidas como estrategias de diversificación de la renda en la pequeña producción agraria. En la discusión desde los objetivos propuestos se han presentadas las experiencias actuales, las potencialidades y los elementos de planeamiento que deben ser añadidos a la actividad turística local para que ella pueda consolidarse efectivamente en favor de los productores de manzana. En las conclusiones se tratan los obstáculos actuales y las perspectivas futuras para el turismo local y regional.

\section{FRUTICULTURA DE CLIMA TEMPLADO Y SISTEMAS AGROALIMENTA- RIOS LOCALIZADOS EN LA REGIÓN SUR DE BRASIL}

En la Región Sur de Brasil se encuentran las áreas más grandes de cultivo de manzanas del país y los estados productores abarcan más del $99 \%$ del volumen recogido. (IBGE, 2011). Las condiciones favorables del medio natural relacionadas con las características del clima subtropical y las altitudes superiores a mil metros de las regiones de montaña resultaron ser las condiciones necesarias para la expansión de la fruticultura de la manzana en esos Estados, siendo las principales variedades cultivadas la Gala, la Fuji y sus mutaciones (EMBRAPA, 2012).

1 «Organizaciones de producción y servicio asociadas a un territorio específico en virtud de sus características y de su funcionamiento». 
La instalación de los campos de manzanas en las áreas más elevadas responde a la dependencia con las bajas temperaturas de estos árboles para la formación de las hormonas localizadas en las yemas, o sea, para que se dé el proceso vulgarmente conocido como «quebra da dormência» ${ }^{2}$. El manzano, de la familia de las Rosáceas, es una planta originaria de Europa que puede llegar a los diez metros de altura, siendo cultivada principalmente en las zonas subtropical y subpolar. El árbol posee un tronco de corteza parda, lisa y copa redonda con flores blancas o rosáceas y aromáticas. El fruto presenta forma de globo con una profunda depresión en el punto de inserción del tallo que la sostiene de las ramas. (Braga et al., 2001, Silva, 2012).

La Región Sur contribuye de manera decisiva a la autosuficiencia en el abastecimiento del mercado consumidor brasileño de manzanas, revirtiendose el cuadro de importación de aproximadamente el 75\% del producto en la década de 1980. (Pereira et al., 2010). La producción regional de manzanas está fundamentada en la fruticultura practicada en pequeñas propiedades, ya que se basa en el uso de la mano de obra familiar. Entretanto, debido a las restricciones de acceso a equipos y tecnologías de procesamiento, la mayor parte de la producción familiar es comercializada a granel. En estos acuerdos, por medio de contratos verticalizados, se establecen patrones de calidad para la determinación del precio de la mercancía, siendo mayoritaria la comercialización de manzana con las agroindustrias alimenticias de bebidas, dulces y embutidos.

La dificultad de posicionar la manzana en el mercado a un precio satisfactorio, sin la intermediación de agentes especuladores, es uno de los desafíos de la agricultura familiar en Brasil. Adicionalmente, la falta de estructuras de producción, conservación y comercialización son puntos críticos de la producción a pequeña escala. La manzana es una fruta perecedera que no puede ser almacenada por un largo período de tiempo, por lo que la condición de seguridad para el comercio de la producción del pequeño productor les obliga a participar en los contratos de integración. Ese tipo de comercio, sin embargo, es extremadamente desventajoso para el pequeño productor rural, habida cuenta que el producto in natura tiene poco valor agregado. Por lo tanto, la ausencia del aprovechamiento de por lo menos parte del montante recogido en la propia propiedad ha ocasionado la dependencia de los productores, de las fluctuaciones de precio dictadas por el mercado mayorista y por las agroindustrias. (Pereira et. al., 2010). En consecuencia, al igual que ocurre en las demás regiones fruticultoras del país, la mayor parte de los productores familiares de esa región trabaja mucho, pero, aún así, está sometida a las más precarias condiciones de vida. No es casualidad que en el 2003 la Microrregión de São Joaquim, una de las más importantes áreas de producción de manzanas del Sur de Brasil, tenía el $55 \%$ de la población en estado de pobreza (Borchardt, 2003). A pesar de eso, el municipio de São Joaquim, el más importante productor nacional de manzana ha sido elegido por el goberno brasileño como uno de los 65 «destinos inductores» del turismo en el país, o sea, entre aquellos que poseen infraestructuras y atractivos calificados para la actividad, caracterizándose como el núcleo receptor y/o distribuidor de flujos turístico (MTUR, 2014). El Municipio de São Joaquim ha sido el único entre los productores de manzana que ha conseguido tal nivel en virtud de la histórica vocación turística local. Aún así, en

2 «quiebra de la somnolencia». 
una encuesta realizada por el Ministério do Turismo en compañía del Serviço Brasileiro de Apoio a Micro e Pequena Empresa (SEBRAE) y la Fundação Getúlio Vargas, entre 12 criterios analizados acerca de la competitividad de los destinos locales en relación a media brasileña, São Joaquim presentó 10 indicadores por debajo de la media nacional. Los indicadores deficientes apuntados por la encuesta fueron: el acceso al municipio, los servicios y equipamientos turísticos, los atractivos turísticos locales, las estrategias de marketing y promoción del turismo local, las políticas públicas, la cooperación regional, la capacidad empresarial además de los aspectos sociales, ambientales y culturales. Los indicadores positivos apenas se notaron en relación a la infraestructura y a la dinámica de la economía local. (FGV/SEBRAE/MTUR, 2010). Considerando esta situación desfavorable, son urgentes e importantes los programas que se propongan dirigir parte de las ganancias obtenidas con la fruticultura regional, a favor de los pequeños productores. En ese contexto, se entiende como deseable la elaboración de estrategias de intervención que busquen el aumento de la renta de los pequeños productores de manzana a partir de la diversificación de las actividades en la propiedad familiar rural.

El proceso de diversificación de las actividades en las propiedades rurales evoca a su vez la noción de pluriactividad, categoría que viene siendo utilizada para describir, así como para señalar la urgencia de un conjunto de nuevas actividades que toman el lugar o se asocian a las actividades agropecuarias en el medio rural (Marafon, 2006). Para Schneider (2003), la pluriactividad es una característica de la organización del medio rural y se refiere a las situaciones en que individuos de la familia con domicilio rural pasan a dedicarse a actividades no necesariamente ligadas a la agricultura. Para los autores, las formas pioneras de la pluriactividad fueron interpretadas por Kautsky (1899) y Chayanov (1925) al referirse al «trabajo rural accesorio» y a las «otras actividades no agrícolas» en el espacio rural. Kautsky trató la cuestión afirmando que la transformación estructural de la agricultura en el capitalismo no elimina necesariamente las pequeñas propiedades ya que ellas desarrollan formas de trabajo accesorias que permitan mantener su reproducción social. De otra manera, Chayanov comprendió que las actividades rurales no agrícolas ubicaban la fuerza de trabajo familiar con el propósito de mantener el equilibrio entre trabajo y consumo, para así garantizar su reproducción.

Entre las actividades «accesorias», las relacionadas con el turismo presentan un potencial para ser implementadas en las pequeñas propiedades rurales de cultivo de manzana y poder contribuir a las políticas de desarrollo territorial local. El concepto de desarrollo territorial aquí utilizado es aquel que involucra la capacidad de cada territorio para aprovechar sus potencialidades y las oportunidades externas (Moraes y Schneider, 2010). Para que el territorio pueda ser utilizado como recurso del desarrollo es necesario, todavía, la «identificación de las aglomeraciones locacionales» para que se puedan conocer sus potencialidades.

Sobre las potencialidades locacionales para el turismo agroalimentario de la manzana, conviene resaltar que los manzanos son cultivados en apenas 71 de los 1188 municipios de la Región Sur de Brasil (figura 1), es decir, en aproximadamente 6\% de ellos. Esos municipios son por lo tanto los que poseen las condiciones adecuadas para la implantación de los manzanos debido a las variables restrictivas relacionadas con las exigencias para el cultivo de la planta. Las restricciones geográficas se deben, sobre todo, a las temperaturas 
comunes en las regiones elevadas de climas templados, a la inclinación y a la calidad de los suelos, factores que combinados favorecen o inhiben la expansión de las áreas de plantación. Es importante recordar que el manzano, siendo de origen subtropical o subpolar es una especie exigente al necesitar horas de fresco para su desarrollo. Las variedades del grupo Gala, por ejemplo, necesitan de aproximadamente setecientas horas de frío, con temperaturas por debajo de $7,2^{\circ} \mathrm{C}$, para tener un rendimiento ideal en la cosecha. Entre las condiciones geográficas restrictivas, la más limitante es la temperatura, seguida de la continentalidad, del relevo, de la nubosidad, de la intensidad y dirección de los vientos y de las características de la propia vegetación. (Braga et al., 2001; Petri et al., 2002; Silva, 2012).

En los municipios productores, el sistema agroalimentario de la manzana está compuesto por propiedades rurales, agroindustrias y cooperativas que seleccionan, procesan y comercializan la fruta y productos derivados de la misma. Junto a estas propiedades están las empresas estatales de investigación y extensión rural, las cuales apoyan a los productores por medio de la investigación y del mejoramiento de los cultivos. Paralelamente a tales segmentos aparece el poder público local que actúa en el sistema por medio de la secretaría municipal de agricultura.

Los mecanismos de promoción de ese sistema agroalimentario como producto turístico, aún así, están por hacerse. Algunas propiedades rurales ya presentan actividades, productos y servicios derivados del cultivo del manzano en condiciones de ser aprovechados como atractivos. Entretanto, se está evaluando, la articulación entre los agentes con el propósito de reforzar el sistema agroalimentario localizado, como motivación del turismo a escala regional con la intención de formular un plan de visitas orientado que incluya los diferentes segmentos productivos.

Los Sistemas Agroalimentarios Localizados (SAL), según Lins (2006), abarcan productores primarios y unidades que benefician productos de origen rural en un mismo espacio geográfico y su localización se encuentra en la intersección del territorio con la cadena de producción-distribución-consumo de un alimento específico. Por ello, el territorio condiciona el funcionamiento de los sistemas agroalimentarios al poner a disposición los activos específicos de que se valen sus agentes. Entre los activos específicos están el conocimiento difundido localmente, la identidad sociocultural y el tejido institucional. Las características más importantes de estos sistemas son, a su vez, los productos alimentarios, la relación con el ambiente, la gestión de los recursos naturales y la vinculación de las actividades agroalimentarias al saber local transmitido por el aprendizaje. (Sautier, 2002; Muchinik, 2002; Moraes y Schneider, 2010).

Los SAL, por su ramificación y estructura en red, presentan la posibilidad de aliarse a las actividades del turismo a medida que ese sector de la economía es igualmente capaz de diversificar y agregar valor a la cadena productiva de alimentos, entre ellos la manzana. Esta fruta, cultivada hace más de cincuenta años en el sur de Brasil, se coloca actualmente como marca de ese espacio regional no solo por la producción agrícola en sí, sino por su significado cultural con referencia a la economía y a la identidad del homem serrano $^{3}$. A pesar de ser un cultivo introducido, habida cuenta que los manzanos no están

3 El término es utilizado, en Brasil, como adjetivo para mencionar a los que viven en áreas de montaña. 
Figura 1

MUNICIPIOS PRODUCTORES DE MANZANA EN LA REGIÓN SUR DE BRASIL

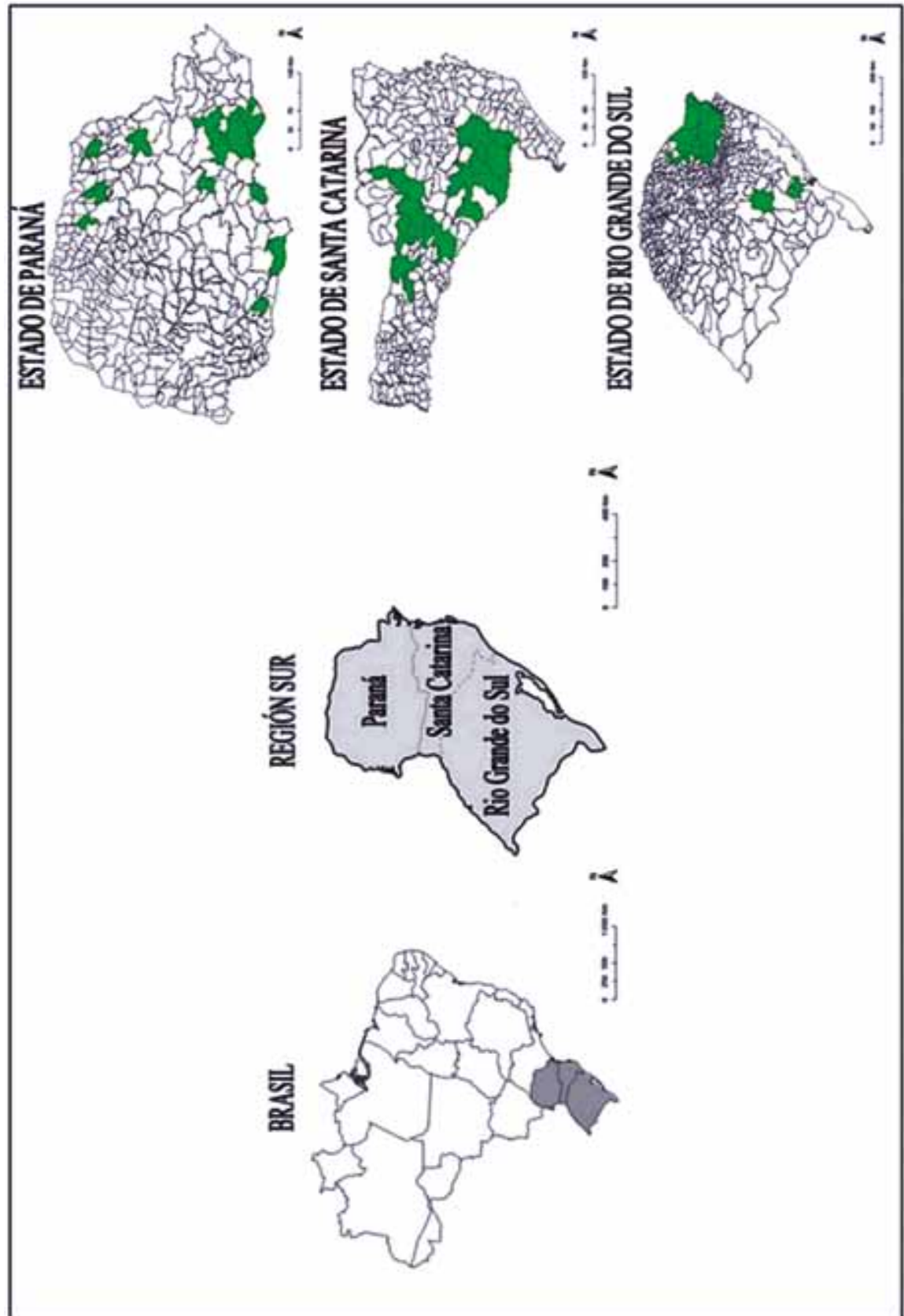

Fuente: Instituto Brasileiro de Geografia e Estatística (2002). Produção Agropecuária Municipal 2011. Rio de Janeiro: IBGE. 
naturalmente distribuidos en las zonas templadas del Hemisferio Sur (Petri et al., 2011; Santos, 1994), la adhesión de los pequeños productores a la actividad y la construcción de un entramado de actores articulados con el procesamiento y comercio de la manzana contribuyó a que ella se convirtiera en un símbolo de la población que vive en las áreas elevadas del Sur de Brasil.

El vínculo entre la manzana y la identidad de la población local es un factor favorable en cuanto se trata de concebir un plan de turismo para el territorio que le otorgue relevancia a un alimento que integre el tejido institucional de diferentes segmentos productivos. En esos términos, conforme menciona Sarrazin (2007), los SAL muestran la forma de organización territorial basada en la espacialización de las actividades agrícolas, de los grupos agroindustriales, de las empresas de servicio, comercialización y restauración, estructuradas en torno a una actividad común relacionada a la producción de alimentos. Cuando están asociados al turismo, los sistemas agroalimentarios pueden incluir además de la red de producción, las actividades de ocio, los atractivos naturales, la gastronomía y los servicios de hospedaje. Esa estrategia de valorización del patrimonio local, por medio del conjunto de bienes y servicios, permite finalmente al territorio desarrollar formas específicas de articulación entre los diferentes segmentos en favor de un plan común de desarrollo.

Para que ese plan tenga éxito, la articulación entre la economía agrícola e industrial debe ser analizada con el fin de incluirlos en negocios y programas de visita. El turismo es, en ese contexto, una forma de agregar otro tipo de valor a las actividades hasta entonces articuladas únicamente en función de la producción de alimentos, una valoración con el fin de generar empleo y renta por medio de la recepción de personas interesadas en experimentar el lugar, conocer y apreciar determinado producto y la comunidad involucrada en su elaboración. El turismo relacionado con los sistemas agroalimentarios tiene todavía condiciones de mejorar la renta de los productores al margen de la cadena principal de valor en determinadas áreas, ya sea como consecuencia de la pérdida de competitividad productiva de las propiedades rurales o como reducción de la capacidad industrial de procesamiento del bien alimenticio. De acuerdo con Briedenhann y Wickens (2004), esta ventaja competitiva ha demostrado ser muy importante cuando se trata de espacios agrarios de regiones empobrecidas en las que la substitución de una cultura agrícola por otra conlleva costos inaccesibles o no se consigue la rentabilidad esperada por el productor. En dichos territorios, el turismo ha surgido como una actividad de revitalizadora del patrimonio cultural y ha contribuido a su conservación por medio de estrategias de uso racional del recurso por parte de la población local.

En la Región Sur de Brasil, el turismo se puede mostrar también como alternativa para la superación de las adversidades materializada en la dependencia de los productores de manzana en relación a las variables negativas impuestas por el mercado fruticultor. Tales variables son las responsables de hacer cada vez más inviable la permanencia de los agricultores en la actividad, como consecuencia de los elevados gastos para el mantenimiento de los pomares, gastos que no coinciden con el precio que se paga por la fruta. Otros eslabones de la cadena productiva de la manzana también sufren con el incremento de la competencia y la caída de precios del producto a partir de la adopción de medidas como el aumento de la importación del alimento y sus subproductos por parte del mercado nacional. Vale destacar que las agroindustrias y cooperativas procesadoras de manzana en 
Brasil están relativamente bien equipadas tecnológicamente, pero a pesar de esto, cuando se considera la cantidad de capital invertido, no consiguen hacer frente a la gran industria alimenticia internacional. En este sentido, el sistema agroalimentario de la manzana, frente a las especificidades productivas, al paisaje, a los productos y a la formación social que presenta, tiene como alternativa, en esa región del país, la estructuración de un proyecto de desarrollo territorial basado en el turismo.

\section{TURISMO AGROALIMENTARIO DE LA MANZANA}

Entre los nuevos usos del espacio rural brasileño están la expansión de las viviendas de segunda residencia, las actividades de conservación de la naturaleza y las actividades de turismo y ocio (Silva, 1997; 2001; Rodrigues, 2001; Schneider, 2006). Estos usos imponen una mayor complejidad en el análisis de las actividades que se instalan en este espacio y, lejos de una uniformidad, cada actividad y recorte regional demandan una perspectiva propia de evaluación de sus efectos en el ámbito territorial. En un país de dimensiones continentales como Brasil, las experiencias de actividades no agrícolas en el espacio rural están relacionadas a variables regionales que implican la forma de fraccionamiento de la tierra y la organización económica de las unidades productivas en el campo. En las regiones donde predominan las extensas propiedades rurales o en las que están alojados los grandes inversionistas, las actividades no agrícolas instaladas en el medio rural se caracterizan por la oferta diversificada de programas de turismo y ocio en establecimientos de un modelo de acomodaciones y atención elevados. Por otro lado, en las regiones con el predominio de propiedades pequeñas y de bajo capital invertido, la introducción de las actividades no agrícolas es generalmente consecuencia del proceso de pérdida de ventajas competitivas de la actividad agropecuaria a las que se dedican los productores rurales y, por lo tanto, es una actividad complementaria con el fin de soportar la disminución de la renta -temporal o permanente- obtenida con la comercialización de los bienes que produce.

Si las variables adversas se ubican casi siempre, como las condiciones objetivas para la diversificación de la renta, en las pequeñas unidades de producción, esto no significa que dichas actividades accesorias sean un problema desde el punto de vista del desarrollo territorial rural. Por el contrario, estas representan una posibilidad concreta de viabilizar económicamente las propiedades rurales. En esa dirección, de acuerdo con Abramovay (1999), no hay razones técnicas para que los pequeños agricultores familiares se alejen del inmenso potencial representado por las nuevas funciones de preservación ambiental y de ocio que el medio rural ofrece para la sociedad.

El espacio rural regional planteado en este trabajo, las áreas de montaña de la Región Sur de Brasil, ha experimentado combinar la actividad agrícola practicada en pequeñas propiedades rurales con otras no agrícolas ligadas al turismo. Ese proceso, iniciado en la década de 1990 y que viene teniendo éxito con las rutas turísticas vitivinícolas, pretende ahora expandir los negocios hacia las propiedades de cultivo de manzana. Con una trayectoria histórica de resistencia a la innovación y a las estrategias de diversificación de renta, los productores sureños de manzana se enfrentan actualmente, con las oscilaciones del precio de la fruta en el mercado, a tener que invertir en actividades de turismo que puedan agregar renta a la familia. 
En esa región, no obstante, la decisión de los productores de entrelazar el cultivo de la manzana con actividades accesorias como el turismo, depende, en general, del retorno económico que la actividad podrá ofrecer. Debido a que los negocios relacionados con el turismo no son conocidos por los productores, la inserción de una rutina de actividades turísticas en la propiedad rural es frecuentemente percibida con desconfianza. Esta está asociada, de un lado, a la inversión de capital en un negocio que, para ellos, no están claras las variables de riesgo. De otro lado, a las implicaciones de las actividades del turismo en el mantenimiento de la rutina de trabajo en la agricultura, hasta el momento la principal ocupación de la propiedad.

El mantenimiento de la agricultura como actividad principal depende así de decisiones subjetivas, aunque legítimas tomadas por los productores para mantener o reducir las horas dedicadas al trabajo en el campo. Es importante atenerse a esta cuestión porque para los productores la actividad agrícola no es solamente un medio de subsistencia, sino también el estandarte de su identidad rural. (Persányi, 1990). La resistencia más fuerte hace referencia al hecho de que las actividades turísticas implican, por su misma naturaleza, el contacto entre agricultores y visitantes, pues en las familias rurales es común cierto recelo y timidez para relacionarse con personas desconocidas. En estos términos, los agricultores «que se beneficiam economicamente com a presença dos turistas não estão precisamente interessados em receber os turistas, mas o dinheiro dos turistas. Os turistas passam a ser um mal necessário. Mal porque sua presença incomoda; necessário porque seu dinheiro faz falta.» (Barreto, 2003: 29). ${ }^{4}$

De esta forma, la inserción del turismo en áreas tradicionalmente de producción agrícola no es fácil. Cabe destacar que este punto es relevante para la comprensión del turismo en el espacio de producción agrícola porque entre los planificadores del turismo es usual no considerar la racionalidad particular del hombre del campo y de la dinámica de la sociedad campesina cuando reflexionan sobre el turismo en dicho espacio. Tal racionalidad imperativa en los valores relacionados con la tierra, la familia y el trabajo rural, podrá parecer muchas veces un contrasentido en términos de opción de inversión para un planificador del turismo.

Es evidente que la actividad accesoria en la pequeña propiedad familiar tiene la posibilidad de convertirse en la actividad principal en el futuro. Esto ocurre, la mayoría de las veces, cuando la actividad agrícola deja de ofrecer los medios de subsistencia que garantizan la supervivencia de los que necesitan de ella o cuando el inmueble rural deja de ser administrado por agricultores. Esa es una condición posible ya sea, por ejemplo, a causa de una restricción para el comercio de determinado bien agrícola debido a la preferencia por la importación o debido al exceso de proveedores de un mismo producto en el mercado. La consecuencia de dicho cambio en la matriz económica de la propiedad rural es, la mayoría de las veces, el abandono de la actividad agrícola como prioritaria, en pro de las más rentables, como las relacionadas al ocio, y la profundización de las necesidades de la planeación estructural como consecuencia de las nuevas funciones atribuidas a la unidad de producción familiar.

4 «que se benefician económicamente con la presencia de turistas no están precisamente interesados en recibir a los turistas, sino el dinero de los turistas. Los turistas se convierten en un mal necesario. Un mal porque su presencia incomoda; necesario porque su dinero hace falta». 
Una cuestión importante a considerar en relación a la planificación de las actividades del turismo en el espacio rural del sur brasileño, se refiere al hecho de que las experiencias de ese tipo están localizadas principalmente en las pequeñas propiedades. Esas propiedades, sin embargo, son aquellas que más sufren con las precariedades estructurales, además de ser las más vulnerables en términos financieros. Se podrían mencionar aquí numerosas causas para dicha vulnerabilidad, pero dos son las más importantes en el contexto de este trabajo: la primera está ligada al fraccionamiento generacional de la propiedad rural, lo que con el tiempo la hace cada vez menos viable para sustentar el número de «bocas» que de ella dependen, una expresión célebre de Ricardo (1987). La segunda causa está relacionada con el tipo de producto agrícola a que se dedica la explotación, habida cuenta que los productores familiares en Brasil están involucrados básicamente con la producción en pequeña escala, especialmente de frutas, como la manzana, y horticultura (hortalizas, verduras y legumbres), es decir, cultivos agrícolas de manejo trabajoso y exigente. Además, dichos cultivos agrícolas son muy vulnerables a las inclemencias climáticas y al control de plagas, además de convivir con las precariedades de precio cuando vendidos, sobre todo, in natura.

En ese contexto, la inserción de algún tipo de actividad turística (de visita, hospedaje, alimentación o servicio) es muchas veces el último recurso para viabilizar la propiedad y lo que ella produce. Sin embargo, la coyuntura desfavorable a causa de la ausencia de conocimientos de los productores sobre el mercado y los negocios del turismo, así como la falta de recursos para inversión, contribuyen casi siempre a hacer más difícil su implementación en el espacio rural. Esta situación es evidente cuando se analiza la fruticultura de clima templado y las propuestas de valorización de la misma a través del turismo en la Región Sur de Brasil.

En esta región, las experiencias de promoción del turismo agroalimentario en los municipios productores han ocurrido a través de pequeños eventos, entre los cuales están las fiestas municipales de la manzana como las de Fraiburgo (SC), Porto Amazonas (PR), Arroio do Padre (RS) y la de São Joaquim (SC). El calendario de eventos en los municipios es una manera de superar los problemas de estacionalidad del calendario turístico a lo largo del año. En estas fiestas hay presentaciones musicales, exposición de productos y platos hechos con manzana, así como de equipos y maquinaria para uso en los manzanares. De acuerdo con Jiménez y De San Eugenio (2009), los eventos representan una importante maniobra de promoción del territorio a la vez que crean imaginarios capaces de producir y consolidar la marca de una ciudad en su conjunto. Incluso según los autores, los eventos contribuyen al crecimiento de la demanda turística, enfatizan los recursos territoriales, potencializan el valor del patrimonio tangible y representan la posibilidad de que el lugar posicione su identidad.

Además de los eventos festivos, en la época de la cosecha de la manzana algunas propiedades promueven el colhe e pague ${ }^{5}$. Las actividades del colhe e pague son similares a los famosos «pesque y pague» que fueron una novedad en Brasil durante la década de 1990. En los «pesque y pague», los turistas pescaban peces en lagos artificiales y al final pagaban por lo que habían pescado. En la actividad del colhe e pague el principio

5 La actividad es conocida por el término pick-your-own en inglés o cueillette à la ferme en francés. 


\section{Figura 2}

COLHE E PAGUE DE MANZANA EN EL MUNICIPIO DE BOMJARDIM DA SERRA, SC.

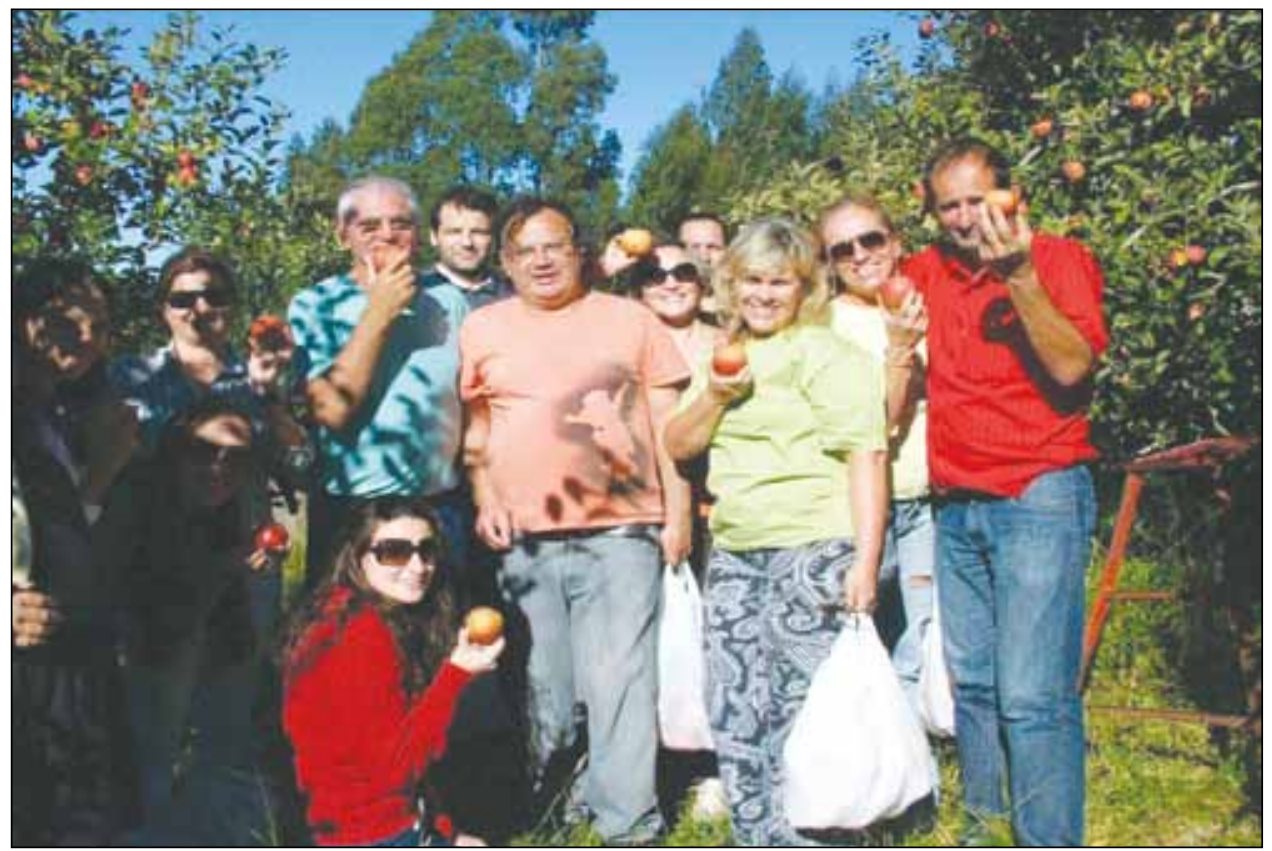

Fuente: Fernando Goulart Rocha. Fecha: Mayo de 2013.

es el mismo: el turista toma una bolsa, recoge las manzanas del manzano y al final paga por lo que recogió (figura 2). Según los productores de manzana, la actividad del colhe e pague se encuentra siempre en relación con el comercio convencional de la fruta en el mercado al de tal, siendo, por lo tanto, una actividad secundaria en la propiedad. ${ }^{6}$

La experiencia de los colhe e pague es aún una actividad aislada, ya que no existe la articulación entre las haciendas que las realizan con los agentes promotores del turismo local. En la misma dirección, no hay una preparación de la familia de agricultores a fin de acoger a los visitantes y revisar informaciones sobre el lugar como, por ejemplo, la historia y el cultivo de la manzana, la inserción de la actividad en la economía regional, las etapas de cultivo del manzano y el destino de la producción. Se considera que durante el período de visita de los campos de manzanos y de la cosecha de las frutas sería apropiado contar con el acompañamiento de los grupos por algún miembro de la familia, ya que la selección de las manzanas se realiza generalmente sin criterios y, con frecuencia, de forma nociva para la planta.

Asociado a los colhe e pague podrían elaborarse productos artesanales en la propiedad (dulces, compotas, mermeladas, té y deshidratados), con el propósito de ser comercializados por los productores. Esos productos permitirían el aprovechamiento de las frutas

6 Información de visita técnica. 
descartadas por el turista y podrían ser vendidos a visitantes y redes de comercio de alimentos. Otra posibilidad es la inserción de esos productos en negocios de alimentación fuera del hogar como restaurantes, cafés, panaderías, confiterías y cafeterías. El escaso aprovechamiento de la materia prima local en los comercios de alimentación es evidente cuando se visitan establecimientos de ese tipo en la región en estudio. Tal como han apontados Schneider y Fialho (2000); Zuin y Zuin (2008); Zuin y Alliprandini (2006), el aprovechamiento no significa apenas la exposición del ingrediente en sí, sino de preparaciones, presentaciones y servicios que identifiquen las costumbres del lugar como patrimonio cultural. Por otro lado, además del período de la cosecha, la observación del florecimiento del manzano, durante la primavera, puede ser incluida como actividad de visita a las propiedades productoras.

En el ámbito agroindustrial, los jugos, fermentados y el aguardiente de manzana (calvados) son las principales bebidas derivadas del procesamiento de la manzana producidas en la Región Sur de Brasil. El comercio de jugos de manzanas nacionales es prometedor, pues el producto es bien aceptado por el consumidor nacional y extranjero. Sin embargo, el mercado de fermentados y destilados es todavía de poca expresión, ya que el consumidor brasileño aún no aprecia la bebida al mismo nivel que la cerveza o el vino. Además de los usos mencionados, la manzana puede ser aprovechada en la elaboración del vinagre y para la extracción de pectina, siendo una de las ventajas del procesamiento agroindustrial de la fruta el aprovechamiento de las unidades que no alcanzan el patrón exigido para consumo. (Rizzon et al., 2005; Arthey y Ashurst, 1997; Varnany Sutherland, 1997).

Los productos agroindustriales derivados de la manzana son actualmente poco aprovechados como atractivos turísticos en comparación a la uva y a los productos resultantes de su procesamiento como jugos y vinos. Una forma de aprovechar el producto de la industria procesadora de la manzana como atractivo turístico es convertirla en parte de los itinerarios de visita en los municipios, integrándola al paisaje y al contexto social del entorno. Conforme Briedenhann y Wickens (2004), el agrupamiento de actividades y atracciones para el diseño de rutas turísticas con señalización adecuada y de fácil acceso es necesario para la planeación del turismo en áreas rurales. La elaboración de rutas turísticas estimula el empresariado local y crea oportunidades de negocios a través de la oferta de servicios opcionales. De esta forma, es un mecanismo de crecimiento económico eficaz verificado en el medio rural de países en desarrollo.

En consecuencia, la instancia gubernamental, ente decisorio de los proyectos de desarrollo, tiene la importante tarea de promover el turismo agroalimentario en los municipios. A pesar de tratarse de un punto recurrente cuando se habla de programas para el turismo en Brasil, no hay duda de que una actividad económica basada en negocios de comercio y servicios, además de pretender incorporar a los pequeños productores familiares, no tiene condiciones de avanzar sin la intervención estatal. Esa intervención debe pasar, entre otras medidas, por una agenda de reuniones con los interesados en invertir en la actividad y en el levantamiento de las prioridades del grupo. Lejos de pretender una homogeneidad de los interesados en relación a las actividades en que buscan invertir, es necesario que haya una evaluación de cómo las actividades propuestas se complementan y de qué manera se pueden articular en la composición de uno o más itinerarios turísticos de visita. 
Para algunas empresas serán necesarias, ciertamente, líneas específicas de financiación para que se viabilicen y, en ese caso, debe haber una evaluación de su prioridad en el contexto del tipo del turismo que se pretende invertir y de las posibilidades de implementarlas a lo largo del tiempo. Lo importante, sin embargo, es que sean aprovechados no solamente los productos más emblemáticos del lugar, sino también del área en que la actividad turística está insertada. Por este motivo, es preciso considerar el paisaje urbano y rural, los atractivos históricos y naturales, así como las narrativas que permitan al turista apropiarse del lugar que experimenta. Una de las ventajas del turismo en áreas rurales, de acuerdo con Wilson et al. (2001), es que este puede desarrollarse con líneas de crédito reducidas, entrenamiento y poco capital. Por lo tanto, los negocios del turismo no exigen prioritariamente costos elevados, pero sí la aplicación de recursos en infraestructuras puntuales de atención al turista y la profesionalización de los agentes locales para la gestión de los negocios familiares.

En otra perspectiva, una tarea importante para la esfera gubernamental es la de agregar y subsidiar mecanismos que den visibilidad a las actividades del turismo localmente instaladas. En este sentido, en la actual coyuntura social de liberación de los medios de acceso a la comunicación y a la información, es extremadamente necesario que los «nuevos» destinos turísticos se promuevan con las herramientas de marketing disponibles. Tal necesidad está fundamentada en la consolidación de un medio técnico que cada vez más logra que la información llegue a las personas con el fin de que conozcan previamente los destinos y atractivos turísticos que desean visitar, especialmente los distantes de los grandes centros.

Es evidente que un programa de marketing que se proponga dar visibilidad al turismo rural debe tener en cuenta el interés de los que buscan dedicar parte de su tiempo libre en ese espacio. Para Mihailovic y Moric (2012), es preciso considerar para el establecimiento de tal programa, el perfil del turista que elige y consume productos turísticos de este tipo. Un rasgo común entre ese tipo de turista, según lo planteado por los autores, es la preocupación por la sostenibilidad. Además, esos turistas asocian lo rural a la idea de «limpieza» y «libertad», además de que están interesados en conocer sobre los lugares que visitan. Estos presupuestos, por lo tanto, no pueden ser descartados en la construcción de una política de marketing para dichas áreas. En otra dirección, las campañas de marketing encaminadas a resaltar el turismo en el medio rural deben resaltar los atractivos turísticos con énfasis en productos regionales a fin de que sea destacada su autenticidad. La estrategia de realzar los productos identitarios de los destinos turísticos sirve tanto para atraer turistas como para apoyar internamente la conservación del patrimonio local.

En la Región Surde Brasil, ese proceso de reconocimiento de la manzana y de las áreas productoras como destino turístico es incipiente en la mayor parte de los estados. En Rio Grande do Sul, el turismo de montaña está actualmente bien organizado, aunque la manzana tenga poco énfasis como producto turístico regional. En Paraná, también es frágil la situación de turismo con relevancia para la producción manzanera. En Santa Catarina, la formación de un proyecto de itinerarios de invierno, con el aprovechamiento de la manzana ya está en curso, siendo seguramente prometedora.

Sin embargo, para que la actividad turística tenga éxito en esa zona del país la cualificación profesional de los pequeños productores rurales y de sus familias hacia el 
turismo es fundamental. Importa notar conforme Schneider y Filho (2000), que parte de los empleos directos producidos por el turismo exigen mano de obra poco calificada en general, lo que casi siempre presenta sueldos bajos para los trabajadores. En este sentido, el turismo puede convertirse en una actividad potencial de duración prolongada en las áreas rurales, aunque la población rural, en general, es poco calificada y muchas veces obtiene ganancias monetarias muy bajas. Sin embargo, a través de la profesionalización, se piensa que se puede elevar el retorno financiero con el turismo por los productores rurales. Para ello, se establecer un patrón de calidad en la recepción de los turistas en los destinos, perfeccionar la gestión de las empresas, organizar estrategias de captación de visitantes y evaluar sistemáticamente los itinerarios implantados. Además de la promoción y de la cualificación profesional, la selección de los atractivos, la construcción y mantenimiento de infraestructura para la recepción de los visitantes, la atención a la hospitalidad y a la calidad en la oferta de servicios son esenciales. (Wilson et al., 2001).

Es oportuno resaltar que la fruticultura de pequeña producción familiar que desea ingresar a las actividades de turismo no se puede desviar de la capacitación técnica con el fin de conocer los medios de agregar valor al bien que producen. En primer lugar, porque el cultivo de frutas es costoso y extremadamente exigente desde el punto de vista de la cantidad de horas de trabajo agregada a la producción. Y luego, porque la fruticultura es dependiente de variables geográficas muy específicas, lo que significa que producir frutas es una actividad con un grado elevado de especialización en áreas de clima templado en Brasil.

Además de productos, pueden estar asociados otros servicios para que la actividad turística pueda ser efectivamente ventajosa para los productores familiares. Entre los servicios que pueden ofrecerse están el hospedaje rural, los cafés coloniales, la conducción por senderos ecológicos así como la visita a atractivos naturales. Todas estas actividades no exigen costos elevados y pueden ser fácilmente incorporados a las demás ofrecidas. A su vez, es perfectamente viable que dichas actividades sean aprovechadas en eventos ya realizados por los municipios a fin de que sean divulgadas, como en las fiestas típicas de la manzana. Esta es una forma relativamente simple de promover la fruticultura regional.

Por último, es necesario destacar que a pesar de que el turismo en las pequeñas propiedades tiene una función muy específica de diversificación de la renta, esto no significa que se coloque aparte de las demás estrategias de promoción de la actividad en escala local. Tratándose del cultivo de la manzana, es importante que los actores involucrados con el procesamiento de la fruta estén articulados de alguna forma con el propósito de convertirla en «bandera» del turismo en las regiones productoras. La articulación de los diferentes actores es fundamental en la formación de una identidad de la fruticultura regional, condición muy viable en las áreas de cultivo de manzanares en el Sur de Brasil.

En ese sentido, es importante pensar el turismo de la manzana como un proyecto colectivo, implementado para generar renta a partir del aprovechamiento del sistema agroalimentar localizado y no con la aparición de acciones individualizadas y desarticuladas entre sí. De esto deriva una planeación para el turismo a partir de estrategias políticas de convencimiento, articulación y evaluación concreta de las posibilidades de inversión con el fin de que se construyan planes y proyectos que puedan ser efectivamente puestos en práctica. 


\section{CONCLUSIONES}

La Región Sur de Brasil, principal productora nacional de manzanas, presenta un potencial para despuntar como destino turístico agroalimentario relacionado al cultivo de la fruta y de los subproductos que de ella se derivan. Las áreas de cultivo de manzano cuentan con el diferencial de estar instaladas en un paisaje de excepción en el territorio brasileño: terrenos por encima de los mil metros de altitud y las únicas donde ocurren precipitaciones de nieve durante la estación de invierno. La producción familiar de manzana en las pequeñas propiedades, el procesamiento de la fruta realizado por las agroindustrias locales y las experiencias como los colhe e pague, son prácticas en curso que apuntan hacia el desarrollo del turismo agroalimentario en las áreas productoras.

El turismo, en el contexto de la pequeña propiedad familiar, se presenta como una estrategia de diversificación de la renta por medio del consorcio entre la actividad agrícola y las relacionadas al ocio. En el Sur de Brasil, la creciente adhesión de propiedades rurales que invierten en algún tipo de actividad con el propósito de complementar el presupuesto familiar ha contribuido para la intensificación de estudios que traten el tema. (Anjos, 2003, Schneider, 2003).

Sin embargo, el escenario actual de las actividades turísticas en esa región presupone el perfeccionamiento de los productos y servicios ofrecidos a los turistas. Por esta razón, se espera una mayor articulación entre los actores involucrados en el cultivo de la manzana y sus beneficios, con el objetivo de que se dé la construcción de rutas turísticas agroalimentarias que tengan como elemento de agregación la pomicultura. Las rutas turísticas agroalimentarias presuponen la creación e implementación de itinerarios que incluyan como atractivos a las propiedades rurales, monumentos históricos, elementos del paisaje natural, productos y servicios artesanales así como agroindustriales que tengan la manzana como materia prima. Así, esta sería una manera de dar visibilidad a la cadena productiva de la fruta en el ámbito regional a través del turismo.

Al mismo tiempo, las rutas turísticas agroalimentarias son un mecanismo que se considera eficiente para pensar la valorización de la manzana de forma colectiva por los actores locales y de superar los obstáculos de las inversiones realizadas de forma aislada. Para que esto ocurra, sin embargo, son imprescindibles los programas de formación profesional a fin de cualificar personas para la elaboración de itinerarios que integren a la comunidad, así como para la recepción y acompañamiento de los visitantes en los sitios de atracción. De otra forma, la cualificación profesional deberá actuar en la concepción de nuevos productos con la finalidad de agregar valor a la fruta, especialmente a aquellas descartadas para consumo in natura.

Además de la cualificación profesional, la organización colectiva de los involucrados con el turismo es fundamental para el éxito de los programas de visita en las propiedades rurales. Aún así, en el momento actual ese es el mayor obstáculo para poder hacer avanzar la actividad considerando que los sujetos apoderados del territorio se muestran, con frecuencia, con poca confianza en cuanto al retorno financiero producto de la actividad turística. En consecuencia, es notoria la desconfianza por parte de una porción de los actores sociales en cuanto a percibir el cultivo de la manzana como un potencial atractivo turístico local. 
Se considera, sin embargo, que la transformación del escenario de organización colectiva desfavorable es posible y está en curso, como revelan las experiencias y las potencialidades del turismo agroalimentario regional. El perfeccionamiento y el mejor desempeño de las propiedades involucradas con este tipo de turismo contribuirán seguramente para el convencimiento de los demás actores, actualmente sin interés en invertir en el sector. Vale recordar que los planes de desarrollo turístico son escasos y recientes en Brasil, especialmente los orientados hacia las áreas rurales, lo que requiere evaluar las condiciones objetivas locales y, al mismo tiempo, levantar propuestas exitosas en otras regiones e países. Entre esas propuestas conviene citar, en Brasil, el Circuito de las Frutas de São Paulo y, en el exterior, las actividades de turismo agroalimentario españolas, entre ellas la Ruta de la Manzana y de la Sidra en el Principado de Asturias.

\section{REFERENCIAS BIBLIOGRÁFICAS}

ABRAMOVAY, R. (1999): «Agricultura familiar e desenvolvimento territorial», Reforma Agrária: Revista da Associação Brasileira de Reforma Agrária, vols. 28, nº 1, 23 y 29. ANJOS, FLÁVIO SACCO DOS. (2003): Agricultura familiar, pluriatividade e desenvolvimento rural no Sul do Brasil. Pelotas: ADUFPEL.

ARTHEY, D. y ASHURST, P.R. (1997): Procesado de frutas. Zaragoza: Acribia.

BARRETTO, M. (2003): «O imprescindível aporte das ciências sociais para o planejamento e a compreensão do turismo», Horizontes. Antropológicos, Porto Alegre, vol. 9, $\mathrm{n}^{\mathrm{o}}$ 20, pp. 15-29. Tourism Management, vol. 25 (2004), pp. 71-79.

BRIEDENHANN, J. y WICKENS, E. (2004): ,Tourism routes as a tool for the economic development of rural areas - vibrant hope or impossible dream?«, Tourism Management, vol. 25, pp. 71-79.

BORCHARDT, I. (2003): Diagnóstico da exclusão social em Santa Catarina. Florianópolis, Instituto CEPA/SC, $235 \mathrm{pp}$.

BRAGA, H.J., JÚNIOR, V. DA S., PANDOLFO, C. y PEREIRA, E. S. (2001): «Zoneamento de riscos climáticos no estado de Santa Catarina», Revista Brasileira de Agrometeorologia, Passo Fundo, vol. 9, n 3, pp. 439-445.

CHAYANOV, A.V. (1925): La organización de la unidad económica campesina. Buenos Aires, Editorial Nueva Visión, 1974.

DÍAZ-BAUTISTA, A. (2001): Efectos de la globalización en la competitividad y en los sistemas productivos locales de México. México, Observatorio de la Economía Latinoamericana.

EMPRESA BRASILEIRA DE PESQUISA AGROPECUÁRIA (2012): Produção integrada de maçãs no Brasil. Extraído em 06/04/2012 de: http://sistemasdeproducao. cnptia.embrapa.br.

FACHINELLO, J.C., PASA, M. DA S., SCHMTIZ, J.D. y BETEMPS, D.L. (2011): «Situação e perspectivas da fruticultura de clima temperado no Brasil», Revista Brasileira de Fruticultura, no 33(spe1), pp. 109-120.

INSTITUTO BRASILEIRO DE GEOGRAFIA E ESTATÍSTICA (2011): Produção Agrícola Municipal. Extraído em 02/06/2013 de: http://ibge.gov.br/cidadesat/topwindow. htm?1 
Food and Agriculture Organization of the United States. (2001). Top production: apples. Food and agricultural commodities. Extraído em 10/06/2013 de: http://faostat. fao.org/site/339/default.aspx.

JIMÉNEZ, M.M. y VELA, J.S.E. (2009): «Identidad territorial y promoción turística: la organización de eventos como estratégia de creación, consolidación y difusión de la imagen de marca del território», ZER: Revista de estúdios de comunicación, vol. 14, no 26, p. 277-297.

KAUTSKY, K. (1899): La cuestión Agraria. Barcelona: Editorial Laia, 1974.

LAVANDOSKI, J. y LANZER, R.M. (2008): A paisagem na rota enoturística Vale dos Vinhedos/RS na perspectiva do visitante. Dissertação de Mestrado, Universidade de Caxias do Sul, 2008. Extraído em 01/06/2013 de http://www.ucs.br/etc/revistas/index. php/dossierosadosventos

LINS, H.N. (2006): «Sistemas agroalimentares localizados: possível «chave de leitura» sobre a maricultura em Santa Catarina», Rev. Econ. Sociol. Rural, Brasília, vol. 44, $\mathrm{n}^{\mathrm{o}} 2$.

LOSSO, F.B., PEREIRA, R.M.F. DO A. (2012): «O desenvolvimento da vitivinicultura e as possibilidades de implantação de roteiros enoturísticos na região de São Joaquim (SC, Brasil)», Revista Brasileira de Pesquisa em Turismo, vol. 6, p. 55-74.

MARAFON, G.J. (2006): «Agricultura familiar, pluriatividade e turismo rural», Campo-Território: Revista de Geografia Agrária, Uberlândia, vol. 1, nº 1, pp. 17-60.

MIHAILOVIC, B. y MORIC, M. (2012): «The role of marketing philosophy in rural tourism», Tourism and Hospitality Management, vol. 18, n 2, pp. 267-279.

PEREIRA, L.B., SIMINI, F.J. y CARIO, S.A.F. (2010): «Evolução da produção de maçã em Santa Catarina: novas estratégias em busca de maior competitividade», Ensaios FEE. Porto Alegre, vol. 31, no 1, pp. 209-234.

MUCHNIK, J. (2002): Les Systèmes Agroalimentaires Localisés: intérêt, approche, interrogations. Exposé Introductif. Colloque. GisSyal, Montpellier.

PERSÁNYI, M. (1990): The rural environment in a post-socialist economy: The case of Hungary. In: Lowe, P.; Marsden, T.; Whatmore, S. Tecnological change and the rural environment: critical perspectives on rural change: series II. London: David Fulton.

MARAFON, G.J. (2006): «Agricultura familiar, pluriatividade e turismo rural», Campo-Território: Revista de Geografia Agrária, Uberlândia, vol. 1, nº 1, pp. 17-60.

PETRI, J.L., LEITE, G.B., COUTO, M. y FRANCESCATTO, P. (2011): «Avanços na cultura da macieira no Brasil», Revista Brasileira de Fruticultura, Jabuticabal, Vol. Especial, pp. 48-56.

PETRI, J.L., PALLADINI, L.A. y POLA, A.C. (2002): «Dormência e indução da brotação da macieira». In: Empresa de Pesquisa Agropecuária e Extensão Rural de Santa Catarina. A Cultura da macieira. Florianopolis: EPAGRI.

RICARDO, D. (1976): Princípios de Economia Política e Tributação. Coleção os pensadores. São Paulo: Abril Cultural.

RIZZON L. A., BERNARDI, J. y MIELE, A. (2005): «Características analíticas dos sucos de maçã gala, Golden delicius e Fuji», Ciência e Tecnologia Alimentar, Campinas, vol. 25(4), pp. 750-756.

RODRIGUES, A. B. (2001): Turismo Rural. São Paulo, Contexto. 
SANTOS, L.W. (1994): «Primórdios da pesquisa com maçã em Santa Catarina», Agropecuária Catarinense. Florianópolis, vol. 7 (3), pp. 20-22.

SARRAZIN, F. (2007): La chambre d'agriculture coordonnatrice, de l'offre du panier de biens et de services agro-touristique landais. VI Rencontres de Mâcon, « Tourismes et territoires » pré-actes, septembre.

SCHNEIDER, S. (2003): «Teoria Social, agricultura familiar e pluriatividade», Revista Brasileira de Ciências Sociais, vol. 18, n 51, p. 99-121.

SAUTIER, D. (2002): Eléments de Perspective. Séance plénière finale. Colloque. Gis Syal: Montpellier, outubro.

SCHEIDER; S. y FIALHO, M.A.V. (2000): «Atividades não agrícolas e turismo rural no Rio Grande do Sul». En Almeida, J.A.; Riedl, M. (Org.) Turismo Rural: ecologia, lazer e desenvolvimento. $1^{\mathrm{a}}$ ed.Bauru: EDUSC, pp. 14-50.

SCHNEIDER, S. (2006): «Turismo em comunidades rurais: inclusão social por meio de atividades não-agrícolas». In: Brasil, Ministério do Turismo. (Org.). Turismo Social: Diálogos do Turismo: Uma Viagem de Inclusão. Rio de Janeiro: Ministério do Turismo/Instituto Brasileiro de Administração Municipal-IBAM, vol. 1, pp. 264-294.

SILVA, J.G. DA (1997): «O novo rural brasileiro», Nova Economia, Belo Horizonte, vol. $7, \mathrm{n}^{\mathrm{o}} 1, \mathrm{pp} .43-81$.

SILVA, J.G. DA. (2001): «Velhos e novos mitos do rural brasileiro», Estudos Avançados, vol. 15(43), pp. 37-50.

SILVA, T.C. (2012): Fruticultura na Serra Catarinense: agroindustrialização da maçã e uva vinífera na Cooperativa Sanjo em São Joaquim, SC, safra 2012. Relatório final de estágio do Curso de Agronomia, Universidade Federal de Santa Catarina, 140pp., mimeo.

VARNAN, A.H. y SUTHERLAND, J.P. (1997): Bebidas: Tecnología Química y Microbiología. Zaragoza: Acribia.

WILSON, S., FESENMAIER, D.R., FESENMAIER, J. y VAN ES, J.C. (2001): «Factors for success in rural tourism development», Journal of Travel Research. vol. 40, pp. 132-138.

ZUIN, L.F.S. y ALLIPRANDINI, D.H. (2006): «Gestão da inovação na produção agropecuária (GIPA)». In: Zuin, L.F.S.; Queiros, T.R. (Org.). Agronegócios: gestão e inovação. 1 ed. São Paulo: Saraiva, vol.1, pp. 252-278.

ZUIN, L.F.S., ZUIN B.P. (2008): «Produção de alimentos tradicionais contribuindo para o desenvolvimento local/regional e dos pequenos produtores rurais», Revista Brasileira de Gestão e Desenvolvimento Regional, vol. 4, nº 1, pp. 109-127. 
